\title{
Message from the BWCCA 2012 International Conference Workshop Co-chairs
}

Welcome to the Workshops of the Seventh International Conference on Broadband and Wireless Computing, Communication and Applications (BWCCA 2012). BWCCA 2012 will be held in conjunction with the Seventh 3PGCIC 2012 International Conference from 12-14 November 2012 at University of Victoria, Victoria, Canada.

This year nine workshops will be held in conjunction with BWCCA 2012 international conference. The workshops are very important part of the main conference and they cover specific topics related to next generation networks, network traffic analysis, sensor technologies, smart environments, complex systems, wireless communication, mobile networks and bio-sensing. BWCCA 2012 workshops are listed in following:

- Fourteenth International Symposium on Multimedia Network Systems and Applications (MNSA 2012)

- Fifth International Workshop on Next Generation of Wireless and Mobile Networks (NGWMN 2012)

- Fourth International Workshop on Network Traffic Control, Analysis and Applications (NTCAA 2012)

- Third International Workshop on Advances in Sensor Technologies, Systems and Applications (ASTSA 2012)

- Third International Workshop on Methods, Analysis and Protocols for Wireless Communication (MAPWC 2012)

- Third International Workshop on Cloud, Wireless and e-Commerce Security (CWECS 2012)

- Second International Workshop on Symbiotic Computing and Multiagent Systems (SCMAS 2012)

- First International Workshop on Bio-Sensing, Processing, Application and Networking (BioSPAN 2012)

These workshops bring to the researchers conducting research in specific themes the opportunity to learn from this rich multi-disciplinary experience. The workshop chairs would like to thank the workshop organizers for their great efforts and hard work in proposing the workshop, selecting the papers, the interesting programs and for the arrangements of the workshop during the conference days.

We hope you enjoy the workshops programs and proceedings.

Akio Koyama, Yamagata University, Japan

Salvatore Vitabile, University of Palermo, Italy

BWCCA 2012 Workshop Co-chairs 